\title{
A new technique of bone cement augmentation via the disc space for percutaneous pedicle screw fixation
}

\author{
Chang Kyu Park, MD, Choon Keun Park, MD, PhD, Dong Chan Lee, MD, and Dong Geun Lee, MD \\ Department of Neurosurgery, The Leon Wiltse Memorial Hospital, Suwon, Republic of Korea
}

OBJECTIVE In elderly patients with severe osteoporosis, instrumented lumbar interbody fusion may result in fixation failure or nonunion because of decreased pedicle screw pullout strength or increased interbody graft subsidence risk. Thus, given its many advantages, percutaneous pedicle screw fixation with cement augmentation can be an effective method to use in elderly patients. The authors report on an easy, safe, and economical technique for bone cement augmentation using a bone biopsy needle inserted into the disc space in 2 osteoporotic patients who were treated with posterior interbody fusion and percutaneous pedicle screw fixation.

METHODS Two elderly patients who complained of back pain and intermittent neurological claudication underwent posterior interbody fusion with percutaneous pedicle screw fixation. After routinely assembling rods on the screws, a bone biopsy needle was inserted into the disc space via the operative field; the needle was then placed around the tips of the screws using fluoroscopic radiography for guidance. Bone cement was injected through the bone biopsy needle, also under fluoroscopic radiography guidance.

RESULTS Both patients' symptoms improved after the operation, and there was no evidence of cage subsidence or screw loosening at the 4-month follow-up.

CONCLUSIONS The indirect technique of bone cement augmentation via the disc space for percutaneous screw fixation could be an easy, safe, and economical method.

http://thejns.org/doi/abs/10.3171/2015.4.SPINE141115

KEY WORDS bone cement augmentation; posterior lumbar interbody fusion; disc space; percutaneous pedicle screw fixation; technique

I NSTRUMENTED lumbar interbody fusion, whether anterior or posterior, immediately produces a biomechanically stable postoperative spine, which facilitates arthrodesis and improves clinical outcomes. ${ }^{1,6}$ In patients with severe osteoporosis, however, instrumented lumbar interbody fusion may result in fixation failure or nonunion because of a decrease in pedicle screw pullout strength or an increase in the risk of interbody graft subsidence, which sometimes requires additional surgery. ${ }^{4,5}$

Many studies have shown that when bone cement is used to augment pedicle screws, it can significantly increase the rigidity, stiffness, and strength of the screw. ${ }^{3,8}$ Various systems and techniques for using cement to augment osteoporotic bone have been developed, such as expandable screws, partially or fully cannulated screws,, 12 and direct ${ }^{2}$ or indirect injections. In recent years, percutaneous pedicle screw fixation, which limits muscular dam- age and intraoperative bleeding, decreases postoperative pain and accelerates postoperative recovery? ${ }^{7}$ However, neither a practical and reliable technique nor an optimal design has been established regarding cement augmentation for percutaneous pedicle screw fixation.

Here we report on an easy and cost-effective technique for bone cement augmentation using a bone biopsy needle inserted into the disc space in 2 osteoporotic patients who were treated with posterior interbody fusion and percutaneous pedicle screw fixation.

\section{Illustrative Cases \\ Case 1}

History and Presentation

This 70-year-old woman presented with back pain and neurogenic intermittent claudication. 


\section{Examination}

Magnetic resonance imaging and dynamic radiography revealed spinal stenosis at L4-5 to S-1 and segmental instability at L4-5. Preoperative bone mineral concentration was $78.36 \mathrm{mg} / \mathrm{ml}$, and the T-score was -4.27 .

\section{Operation and Postoperative Course}

The patient underwent posterior interbody fusion with percutaneous screw fixation at L4-5 and simple canal decompression at L5-S1. After routinely assembling rods on the screws, bone cement augmentation was performed by inserting a bone biopsy needle into the disc space to prevent cage subsidence and screw loosening. Postoperatively, the symptoms had diminished 4 months later; there was no evidence of cage subsidence or screw loosening on the follow-up plain radiographs.

\section{Case 2}

History and Presentation

This 68-year-old woman presented with severe back pain and radiating pain in both legs with neurogenic intermittent claudication.

\section{Examination}

Magnetic resonance imaging and dynamic radiography revealed spondylolisthesis and spinal stenosis at L4-5 and acute compression fractures at L-1 and L-5. Preoperative bone mineral concentration was $31.92 \mathrm{mg} / \mathrm{ml}$, and the Tscore was -6.12 .

\section{Operation and Postoperative Course}

The patient underwent posterior interbody fusion with percutaneous screw fixation at L4-5 and percutaneous vertebroplasty at L-1. After routinely assembling rods on the screws, bone cement augmentation was performed by inserting a bone biopsy needle into the disc space to prevent cage subsidence and screw loosening. Postoperatively, the symptoms had diminished, and 4 months later, there was no evidence of cage subsidence or screw loosening on the follow-up plain radiographs (Fig. 1).

\section{Surgical Technique}

The patient was placed prone after general anesthesia was induced. The level of skin incision was confirmed with C-arm fluoroscopy, and a midline skin incision was made, although we limited the size of the skin incision for only lamino-facetectomy and cage insertion to minimize blood loss, muscle damage, and operative time. The size of the midline skin incision was about $4-5 \mathrm{~cm}$. We performed posterior lumbar interbody fusion, but saved the posterior ligamentous complex, which included the supraspinous ligament, interspinous ligament, and spinous process, to minimize both bone destruction and postoperative adjacent segmental instability.

After the discectomy, we prepared the endplate with a curette, and cages were inserted at the interbody space. We inserted percutaneous pedicle screws using fluoroscopic radiography (Siemens) for guidance before skin closure. After routinely assembling rods on the screws, a bone bi-

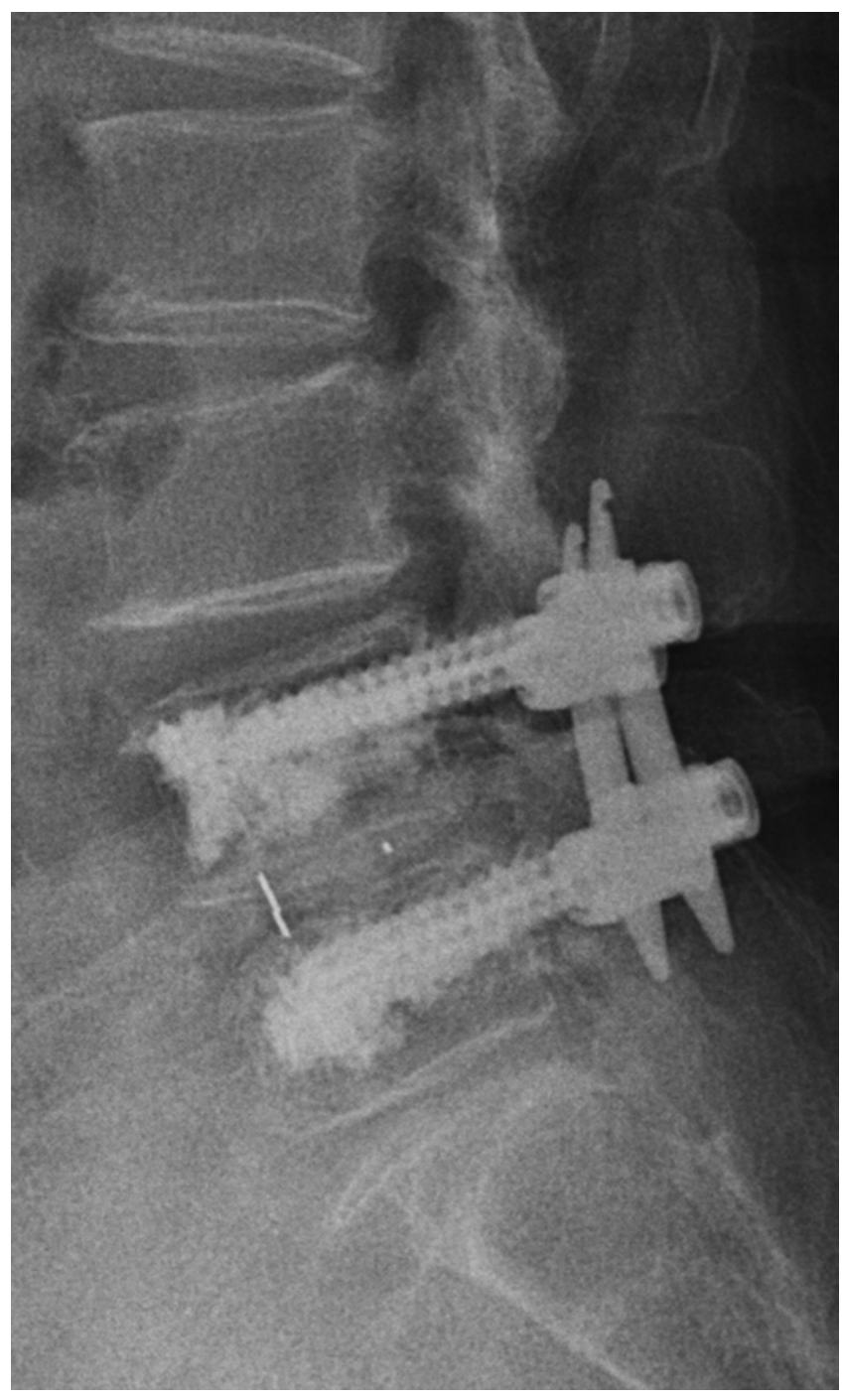

FIG. 1. Case 2. Plain radiograph, lateral view, shows no cage subsidence or screw loosening at 4 months postoperation.

opsy needle (Angiotech Inc.) was inserted into the disc space via the operative field and placed around the tips of the screws; fluoroscopic radiography was used for guidance. We then injected a mixture containing $70 \%$ polymethylmethacrylate (PMMA) powder and 30\% sterilized barium powder (Elmdown Inc.) via the bone biopsy needle. As we adjusted the needle's angle of insertion, bilateral cement augmentation was achieved using a unilateral approach. When we performed bone cement augmentation on the caudal body, a bone biopsy needle was inserted into the upper posterior surface of the caudal vertebral body via the operative field and placed around the tips of the screws (Fig. 2). When the needle is withdrawn slowly, it is possible for bone cement to inject to about one-third the screw length and the midportion of the vertebral body.

\section{Discussion}

Spine surgeons are more and more concerned about the aging spine, and they have to deal with patients with a sig- 


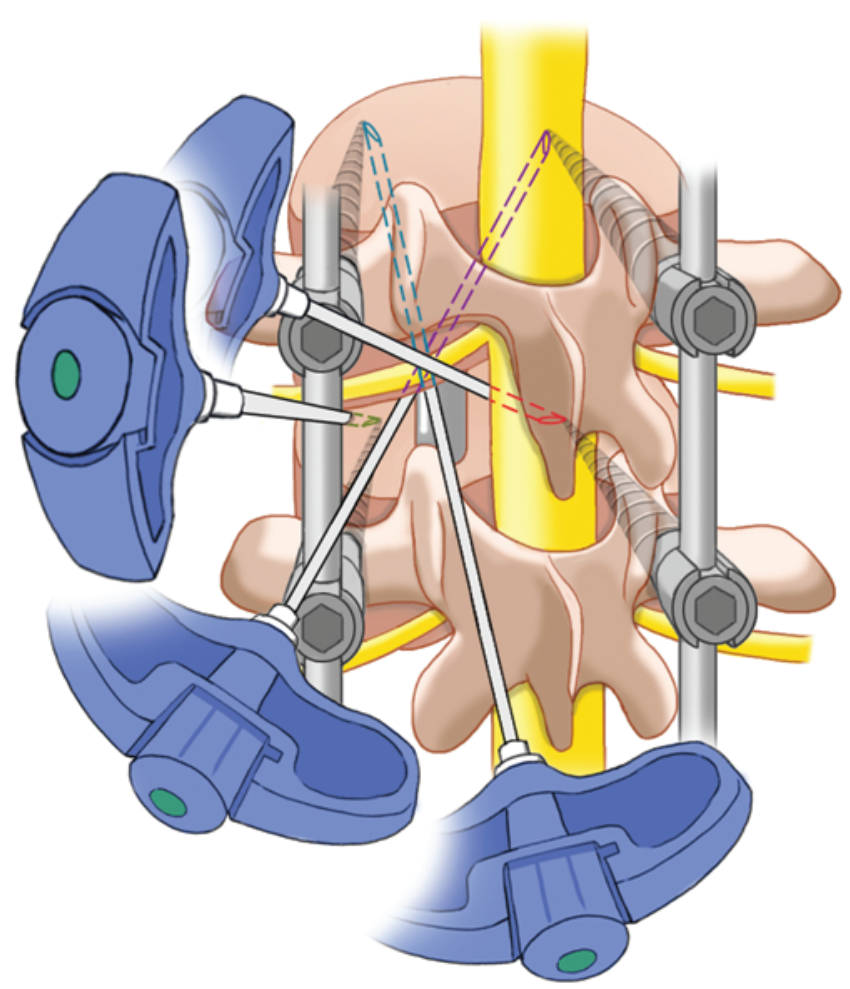

FIG. 2. Schematic demonstrating the bone cement augmentation technique with the bone biopsy needle inserted into the disc space. Copyright Dong Geun Lee. Published with permission. Figure is available in color online only.

nificant loss of bone stock. Performing an arthrodesis in these patients can be difficult because of the osteoporosis and comorbidities that increase complication rates. ${ }^{10}$ Various techniques to reduce these operative risks have been described. Among them, a percutaneous pedicle screw fixation can be a valuable option because it leads to a decrease in surgical time, blood loss, and infectious complications. These techniques may help reduce muscle trauma and promote a quicker postoperative recovery.

On the other hand, percutaneous pedicle screw fixation by itself is not the answer to severe osteoporosis or a significant loss of bone stock that can lead to screw pullout or pedicle fracture. A combination of percutaneous pedicle screw fixation and cement-augmented screws can therefore be a valuable option in the management of these fragile patients. ${ }^{7}$ Several previous biomechanical studies have reported that cement-augmented screws using PMMA have higher pullout strength than conventional screws, 3,11 while there is no evidence with respect to increasing solid bony arthrodesis.

A recent study reported that percutaneous cement-augmented screw fixation with cannulated screws was effective in fractures of the aging spine. ${ }^{7}$ Cannulated percutaneous pedicle screws may be useful for long-level fixation because it is possible to align the screw heads before bone cement is injected.

$\mathrm{Kim}^{6}$ suggested anterior bone cement augmentation in anterior lumbar interbody fusion and percutaneous pedicle screw fixation in patients with osteoporosis. The loca- tion of the cement mass is limited to the middle one-third of the vertebral body on both anteroposterior and lateral images to avoid the trajectory of the pedicle screw. However, this is done to prevent cage subsidence rather than to increase the stiffness and strength of the screw.

We think that our bone cement augmentation technique has several merits. First, it is an easy procedure to practice and is similar to percutaneous vertebroplasty. In cases of multilevel posterior interbody fusion, it is much easier to perform than direct injection with a biopsy needle. Second, the bone cement augmentation technique is more economical than a technique using cannulated percutaneous screws. When we perform a 1-level posterior interbody fusion, the cost of the screw set including the cage plus 4 bone biopsy needles is about $\$ 1752$ at a minimum, whereas the cost of a cannulated percutaneous screw set is about $\$ 3306$ at a minimum in Korea. Third, if bone cement leaks into the epidural space during the injection, it would be easily noticed and handled via the operative field. Fourth, the technique is helpful not only in preventing cage subsidence but also for increasing the stiffness and strength of the screw if bone cement is placed around the screws properly as in a direct injection.

Some may think that the T-scores of -4.27 and -6.12 in our cases are too low to attempt fusion surgery. Including in these cases, we used quantitative computed tomography (QCT) to measure bone mineral density. In the spine, QCT measures the bone mineral density of only the spongy interior bone and does not assess the dense cortical bone that forms the exterior walls of the vertebrae. Consequently, the results of bone mineral density quantification depend on the method by which the region of interest is evaluated. Dual-energy x-ray absorptiometry (DEXA) is aimed at the patient's bones, including cortical bone, while soft tissue absorption is subtracted out. For this reason, the T-score obtained by QCT was a little lower than that obtained by DEXA. We think that the actual Tscores of these patients are a little higher than the T-scores obtained by QCT. Nevertheless, we accept that achieving solid fusion is not easy considering the low T-scores of the patients.

Limitations of this report are its small sample size and insufficient follow-up period for judging long-term clinical and radiological outcomes. Therefore, more patients and longer follow-up times are necessary before definitive conclusions can be reached. Moreover, this cement augmentation technique is only feasible for patients treated with an open decompression or posterior lumbar interbody fusion.

\section{Conclusions}

The indirect technique of bone cement augmentation through the disc space for percutaneous screw fixation is potentially an easy, safe, and economical method to use in elderly patients who have osteoporosis.

\section{Key Points}

1) Percutaneous pedicle screw fixation with cement augmentation can be a valuable option in the management of elderly patients with osteoporosis. 
2) Performing percutaneous pedicle screw fixation using the general indirect-injection technique via a biopsy needle is difficult to do and time-consuming.

3) The direct-injection technique may lead to screw failure if the screws are not exactly lined up.

4) The indirect technique of bone cement augmentation via the disc space for percutaneous screw fixation is an easy, safe, and economical method.

\section{References}

1. Brantigan JW, Steffee AD: A carbon fiber implant to aid interbody lumbar fusion. Two-year clinical results in the first 26 patients. Spine (Phila Pa 1976) 18:2106-2107, 1993

2. Chang MC, Liu CL, Chen TH: Polymethylmethacrylate augmentation of pedicle screw for osteoporotic spinal surgery: a novel technique. Spine (Phila Pa 1976) 33:E317-E324, 2008

3. Cook SD, Salkeld SL, Stanley T, Faciane A, Miller SD: Biomechanical study of pedicle screw fixation in severely osteoporotic bone. Spine J 4:402-408, 2004

4. Halvorson TL, Kelley LA, Thomas KA, Whitecloud TS III, Cook SD: Effects of bone mineral density on pedicle screw fixation. Spine (Phila Pa 1976) 19:2415-2420, 1994

5. Jost B, Cripton PA, Lund T, Oxland TR, Lippuner K, Jaeger $\mathrm{P}$, et al: Compressive strength of interbody cages in the lumbar spine: the effect of cage shape, posterior instrumentation and bone density. Eur Spine J 7:132-141, 1998

6. Kim Y: Finite element analysis of anterior lumbar interbody fusion: threaded cylindrical cage and pedicle screw fixation. Spine (Phila Pa 1976) 32:2558-2568, 2007

7. Pesenti S, Blondel B, Peltier E, Adetchessi T, Dufour H, Fuentes S: Percutaneous cement-augmented screws fixation in the fractures of the aging spine: is it the solution? Biomed Res Int 2014:610675, 2014

8. Pfeifer BA, Krag MH, Johnson C: Repair of failed transpedicle screw fixation. A biomechanical study comparing polymethylmethacrylate, milled bone, and matchstick bone reconstruction. Spine (Phila Pa 1976) 19:350-353, 1994
9. Piñera AR, Duran C, Lopez B, Saez I, Correia E, Alvarez L: Instrumented lumbar arthrodesis in elderly patients: prospective study using cannulated cemented pedicle screw instrumentation. Eur Spine J 20 (Suppl 3):408-414, 2011

10. Ponnusamy KE, Iyer S, Gupta G, Khanna AJ: Instrumentation of the osteoporotic spine: biomechanical and clinical considerations. Spine J 11:54-63, 2011

11. Sarzier JS, Evans AJ, Cahill DW: Increased pedicle screw pullout strength with vertebroplasty augmentation in osteoporotic spines. J Neurosurg 96 (3 Suppl):309-312, 2002

12. Wu ZX, Gao MX, Sang HX, Ma ZS, Cui G, Zhang Y, et al: Surgical treatment of osteoporotic thoracolumbar compressive fractures with open vertebral cement augmentation of expandable pedicle screw fixation: a biomechanical study and a 2-year follow-up of 20 patients. J Surg Res 173:91-98, 2012

\section{Disclosure}

The authors report no conflict of interest concerning the materials or methods used in this study or the findings specified in this paper.

\section{Author Contributions}

Conception and design: DG Lee, Choon Keun Park. Acquisition of data: DG Lee, Chang Kyu Park. Analysis and interpretation of data: DG Lee, Chang Kyu Park. Drafting the article: Chang Kyu Park. Critically revising the article: DG Lee, Choon Keun Park, DC Lee. Reviewed submitted version of manuscript: DG Lee, Choon Keun Park, DC Lee. Approved the final version of the manuscript on behalf of all authors: DG Lee. Study supervision: DG Lee, Choon Keun Park, DC Lee.

\section{Correspondence}

Dong Geun Lee, Department of Neurosurgery, The Leon Wiltse Memorial Hospital, Gyeongsudaero 437, Paldal-Gu, Suwon, Gyeonggi Province 442-070, Republic of Korea. email: vitamine-lee@hanmail.net. 